\title{
Toward Engineering Synthetic Microbial Metabolism
}

\author{
George H. McArthur IV and Stephen S. Fong \\ Department of Chemical and Life Science Engineering, Virginia Commonwealth University, 601 W. Main Street, \\ Richmond, VA 23284-3028, USA
}

Correspondence should be addressed to Stephen S. Fong, ssfong@vcu.edu

Received 15 August 2009; Accepted 9 October 2009

Academic Editor: Hal Alper

Copyright ( $) 2010$ G. H. McArthur IV and S. S. Fong. This is an open access article distributed under the Creative Commons Attribution License, which permits unrestricted use, distribution, and reproduction in any medium, provided the original work is properly cited.

\begin{abstract}
The generation of well-characterized parts and the formulation of biological design principles in synthetic biology are laying the foundation for more complex and advanced microbial metabolic engineering. Improvements in de novo DNA synthesis and codonoptimization alone are already contributing to the manufacturing of pathway enzymes with improved or novel function. Further development of analytical and computer-aided design tools should accelerate the forward engineering of precisely regulated synthetic pathways by providing a standard framework for the predictable design of biological systems from well-characterized parts. In this review we discuss the current state of synthetic biology within a four-stage framework (design, modeling, synthesis, analysis) and highlight areas requiring further advancement to facilitate true engineering of synthetic microbial metabolism.
\end{abstract}

\section{Introduction}

As with its sister field of systems biology, synthetic biology is perhaps best described not by what you do, but how you do it. From this perspective, one way of summarizing synthetic biology is by its intended goal of making biological systems explicitly tractable through careful modularization of biology [1]. In this vein, the core emphases in synthetic biology are synthesis, abstraction, and standardization of biological components [2]. Synthesis refers to the generation of modular, reusable biological parts (normally as DNA). Abstraction refers to the implementation of synthesized parts by function rather than composition (e.g., "this is a promoter" rather than "this is ttgacagctagctcagtcctaggtataatgctagc") and can facilitate the design of more complex biological devices and systems. One of the hallmarks of this approach is the push for standardization where all synthesized parts and devices are characterized, reproducible, and interchangeable.

Although nascent, the approach employed in synthetic biology is already making significant contributions in areas within biological engineering and basic biological research by providing a rigorous framework for building biological systems from the ground-up [3]. Progress in synthetic biology is providing the concepts and tools to develop biological engineering as an application of biology, just as chemical engineering is an application of chemistry. Although the scope of applied synthetic biology is wide, including areas that are "beyond the bioreactor" such as bioremediation, agriculture, and human health [4], it is apparent that many of the applications of synthetic biology that will materialize in the near future are in metabolic engineering [5]. In this context, the synthetic biology approach aims to generate scalable, reusable genetic parts for controlling genetically encoded unit operations (e.g., enzymes, transporters), for the predictable design and construction of metabolic pathways [6].

Since its inception, made possible by the advent of recombinant DNA technology and the polymerase chain reaction (PCR), the goal of metabolic engineering has been to optimize cellular metabolism for a particular process application-often through extensive genetic modification. While research in metabolic engineering has continued to advance with a growing number of genetic, analytical, and computational tools [7], the application of synthetic biology to metabolic engineering has the potential to create a paradigm shift [8]. Rather than starting with the full complement of components in a wild-type organism and piecewise modifying and streamlining its function, metabolic 


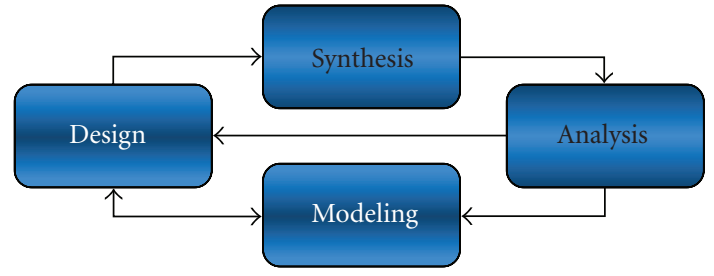

Figure 1: Synthetic biology four-step engineering workflow. The design and modeling steps (white text) are computational in nature, whereas the synthesis and analysis steps (black text) are experimentally-based. The workflow presents the relationships between the individual steps as well as a logical progression using a synthetic biology framework to generate a product that meets determined specifications.

engineering can be attempted from a bottom-up, parts-based approach to design by carefully and rationally specifying the inclusion of each necessary component. In this way, the metabolic engineering process becomes uniform for any goal and eliminates laborious cycles of trial and error.

In this review, we discuss synthetic biology as it applies specifically to metabolic engineering applications. For the purposes of this discussion, we are presenting the typical workflow of synthetic biology within a four step framework (Figure 1). As depicted in the flow diagram, the typical stages involved are: design, modeling, synthesis, and analysis. The workflow is often highly cyclical, although progress is being made on moving from iterative biological design to more predictable, directed design. In the following sections, we discuss recent progress and current challenges in applying synthetic biology to metabolic engineering for each of the four workflow steps (design, modeling, synthesis, and analysis) in general and specifically at the level of genetic parts and metabolic pathways. While synthetic biology applies to all levels of biological organization, we are delineating parts (individual functional units such as promoters, ribosomal binding sites, protein coding sequences, and terminators) from pathways (part-based systems) as there are often different considerations or methodologies employed at these levels.

\section{Design}

Imagine an architect preparing blueprints for someone's dream house. In the design process, the architect will largely focus on including all of the desired functional and aesthetic components but there will also need to be some consideration given to standard safety and logistical requirements. A draft blueprint can be generated to best satisfy the functional and logistical requirements and after the design is approved, the materials needed for construction can be purchased. In this scenario, the design and construction (synthesis) phases are decoupled due to the availability of standard, readily available building materials. Currently, the design and synthesis of biological systems are not decoupled. For example, the construction of metabolic pathways from genetic parts first requires a collection of wellcharacterized parts, which do not yet exist. This shortcoming is beginning to be addressed by the synthetic biology community through the development and compilation of standard biological building blocks such as those found in the Registry of Standard Biological Parts. Although virtually nonexistent now, the future availability of modular, functionally interoperable genetic parts that are carefully characterized will allow biological design to focus more exclusively on functional, aesthetic, and logistical concerns independent of construction concerns.

2.1. Parts. In designing individual biological parts, the base-by-base content of that part (promoter, ribosomebinding site (RBS), protein coding region, terminator, etc.) is explicitly dictated. Rules and guidelines for designing genetic parts at this level are just beginning to be established [9]. In particular, a major consideration in designing protein-coding parts is codon optimization, encoding the same amino acid sequence with an alternative, preferred nucleotide sequence. Although a particular sequence, when expressed, may be theoretically functional, its expression may be far from optimal or even completely suppressed due to codon usage bias in the heterologous host [10]. Although the design principles for expressing exogenous genes have not been perfected, existing codon optimization strategies are used routinely to enhance pathway performance. Codon optimization of coding sequences can be achieved using freely available algorithms such as Gene Designer, standalone software made by DNA2.0. In addition to codon optimization, other considerations such as compliance with standard assembly requirements and part-specific objectives including activity or specificity modifications are often considered. For example, the BioBrick methodology requires that BioBrick parts exclude four standard restriction enzyme sites, which are reserved for use in assembly [11].

Extensive collections of parts can be generated by using a naturally occurring part as a template and rationally modifying it to create a library of that particular genetic part. Significant progress in this area has been recently demonstrated for promoters and RBSs [12, 13]. The work by Ellis et al. documents the generation and characterization of two promoter libraries that can be used to tune network behavior a priori by fitting mathematical promoter models with measured parameters [12]. This model-guided design approach limits system variability, increasing predictability and decreasing the time spent on combinatorial system construction, testing and debugging. Noisy or leaky promoters can complicate system design by adding variability. In these cases, finer control over expression can be established by weakening the binding strength of the downstream RBS [14] or by using two promoter inputs to drive transcription of an output via a modular AND gate [15].

In addition to increasing the number of parts within existing families (e.g., constitutive promoters), there is an opportunity to develop entirely new parts families such as those used to build RNA devices that can regulate expression posttranscriptionally [16]. Win and Smolke developed RNA 
devices that incorporate RNA aptamers for sensing small molecules, transmitter sequences that transmit information by way of a conformational change, and actuators such as hammerhead ribozymes that may render the mRNA untranslatable depending on the state of the sensor and transmitter [17]. The benefit of these devices is that they are composed of modular parts and are completely scalable, since RNA aptamers can theoretically be designed to sense any small molecule ligand [18].

2.2. Pathways. Rational pathway design can be thought of as mixing and matching well-known, modular parts and modulating gene expression through various control mechanisms. One of the primary motivations behind the attempt to achieve fine control of gene expression in the context of metabolic engineering is simply to balance and optimize flux through a desired pathway [19]. Thus, design at the pathway-level is not only concerned with including the necessary parts, but also with controlling the expressed functionality of those parts (e.g., a specific gene may be used in different pathways, the level of required expression may differ based upon the context).

Parts-based synthetic metabolic pathways will require tunable control, just as their natural counterparts which often employ feedback and feedforward motifs to achieve complex regulation [20]. The temporal and spatial dynamics necessary for life (e.g., diffusion, evolution, growth/death) make it difficult to achieve this goal. For example, biological signals must diffuse throughout the cell until it is received by the signal's receptor, unlike an electrical signal that is insulated and directed along a path. Therefore, information transfer in biological systems is intrinsically noisy, an issue that has been reviewed and continues to be explored $[21,22]$. This is the case for gene regulation at the transcription level in which there is a DNA-protein interaction between regulatory proteins such as the LacI repressor and its operator site, lacO. Although a handful of operator-regulator pairs have been used successfully to construct simple genetic circuits $[23,24]$, more complex networks will likely require a large repetoire of distinct regulatory elements to eliminate molecular cross-talk. Developing regulatory parts such as promoters, operator-regulator pairs, ribosome binding sites (RBSs), and riboswitches is a proven way to tackle this problem [25]. Table 1 lists a sampling of regulatory parts for control of various biological processes involved in gene expression.

Another approach, based on well-studied natural systems [26], was recently demonstrated by Dueber et al. to complement these methods. This elegant approach uses protein scaffolds that bear modular interaction domains to physically link pathway enzymes that have been tagged with the appropriate corresponding peptide ligands [27]. Not only does enzyme colocalization limit the loss of intermediates to competing pathways, it also enables the direct control of metabolic flux by adjusting the number of interaction domains on the scaffold thereby adjusting the enzyme complex composition.
Using a synthetic biology approach, the design of DNA sequences encoding metabolic pathways (e.g., operons) should be relatively straightforward. However, the prerequisite collection of modular genetic parts has not yet been developed, but progress has been made [28]. Synthetic scaffolds and well-characterized families of regulatory parts have emerged as powerful tools for engineering metabolism by providing rational methodologies for coordinating control of multigene expression as well as decoupling pathway design from construction. This approach begins to solve the "impedance problem" by providing component variants with covering a range of input/output transfer functions, allowing pathway tenability [29]. Pathway design should not overlook the fact that exogenous pathways interact with native cellular components and have their own specific energy requirements. Therefore, modifying endogenous gene expression (e.g., gene knockouts, knockdowns, overexpression) may be necessary in addition to balancing cofactor fluxes (e.g., ATP, $\mathrm{NADH}$ ) and installing membrane transporters [30]. Figure 2 illustrates how well-characterized parts can be composited to form functional devices or modules that can then be used to build complex systems, including metabolic pathways.

\section{Synthesis}

Just as cars are routinely built from modular components in assembly lines, rapid, modularized construction of microbial chemical factories is on the horizon. Enabling this manufacturing process is DNA synthesis technology, the chemical fabrication of gene- and even genome-length DNA molecules. For example, putative genes identified computationally in a metagenomics study need not be isolated and cloned; the sequence information can be outsourced to synthesis companies as Bayer et al. recently reported [31]. However, protocols exist to synthesize DNA in laboratories using common equipment and techniques [32]. This capability opens many opportunities for metabolic engineers. First of all, the convenience of this approach over traditional cloning allows for the systematic generation of genetic part variants such as promoter libraries (see Section 2). Secondly, it provides a practical way for eliminating restriction sites or undesirable RNA secondary structures and to codon optimize genes for the expression in heterologous hosts. Thirdly and perhaps most importantly, the generation of biological building blocks will allow for the decoupling of construction from design.

3.1. Parts. Generating genetic parts is as easy as emailing the part's nucleotide sequence to a DNA synthesis company such as DNA2.0, GENEART, or Genscript. Depending on the length and complexity of the part, it could take as little as a week to synthesize and ship. Although synthesis technology is available for individual laboratories, the time and money required for equipment upkeep may not make investment worthwhile, especially with commercial synthesis prices steadily dropping. Still, the cost of DNA synthesis is relatively high and can be a prohibitive factor for some projects. It is anticipated that DNA synthesis technology 


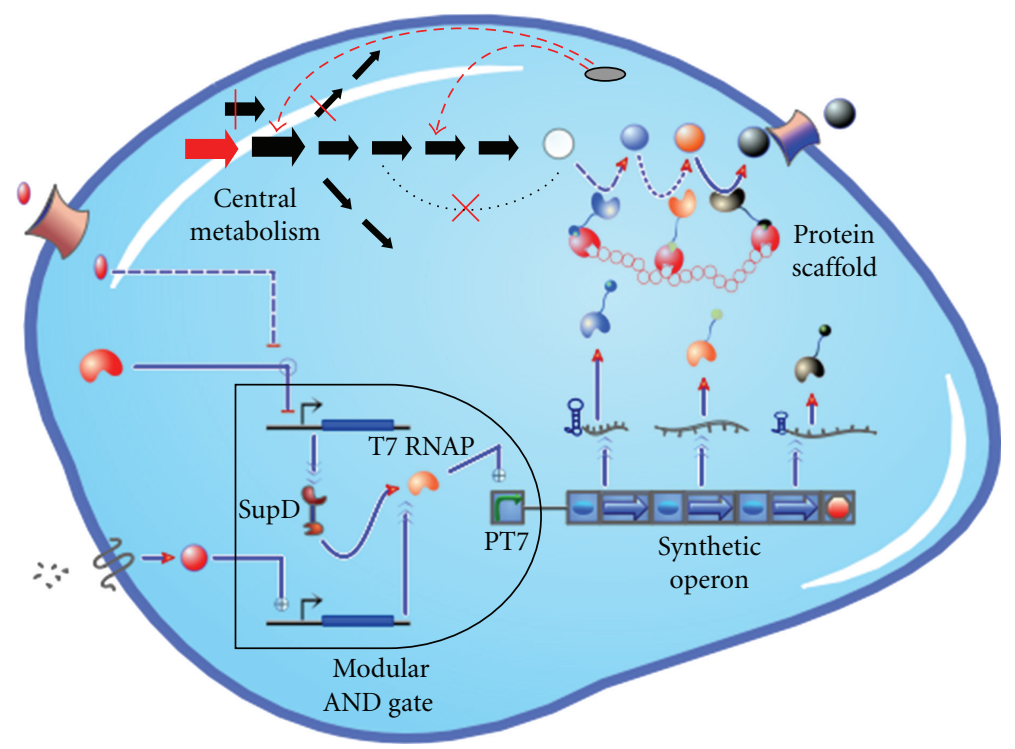

FIGURE 2: Cartoon of an engineered cell. Four distinct strategies have been combined to illustrate a synthetic biology approach to metabolic engineering. The engineered pathway is encoded by a synthetic operon designed from standard parts/components including tunable intergenic regions (TIGRs) that provide differential control over mRNA stability [41]. An upstream device/module, a transcriptional modular AND gate acts as a master regulator by requiring two separate inputs to turn on expression of the pathway [15]. The enzymes have been tagged with a peptide ligand that binds them to a synthetic protein scaffold, forming a complex of colocalized pathway enzymes [27]. The central metabolism of the host/chassis has been modified to interface with the engineered system/network, providing a sufficient flux of necessary precursors and cofactors as well as increased transporters to facilitate product secretion [7]. This image was generated in TinkerCell.

will follow in the footsteps of DNA sequencing technology where research and improvements have decreased costs by orders of magnitude. Czar et al. provide an excellent review of synthesis technologies and strategies, including those that enable in-house synthesis [33].

3.2. Pathways. The ability to make large changes to DNA molecules has resulted in standardized methods for assembling basic genetic parts into larger composite devices, which facilitate part-sharing and faster system-level construction. For example, the Registry of Standard Biological Parts supports the use of the BioBrick methodology to assemble parts into devices and devices into systems. The restriction sites flank BioBrick parts (two serve as the prefix and the other two as the suffix) allowing for restriction-ligation all the while maintaining the same restriction sites. A standardized assembly method, 3A BioBrick assembly, enables a threeway ligation of two insert parts into a plasmid backbone and eliminates the need to gel-purify digested parts and plasmid backbones [11]. This approach can be automated using programmable liquid-handling robots, providing a scalable assembly process that can be used to quickly build systems from existing genetic components. In this respect, highthroughput pathway assembly is more easily implemented in individual laboratories than de novo DNA part synthesis, but there remains the option to outsource this aspect of synthesis through commercial avenues as well.

Other approaches based on type II restriction enzymes, such as Golden Gate Shuffling, provide ways to assemble many more components together in one step [45]. A similar one-step assembly approach, circular polymerase extension cloning (CPEC), avoids the need for restriction-ligation or single-stranded homologous recombination altogether. This scalable, sequence-independent cloning technology works by extending overlapping sequences between the part and the plasmid backbone (e.g., BioBrick prefix and suffix), just as polymerase extension occurs in PCR, resulting in a complete circular plasmid [46]. Not only is this useful for cloning single genes, but also for assembling parts into a larger sequences encoding entire metabolic pathways and for generating combinatorial part libraries.

Multiplex automated genome engineering (MAGE) was recently introduced as another scalable, combinatorial method for producing large-scale genomic diversity [47]. This approach makes chromosomal modification easier by simultaneously mutating target sites across the chromosome. In this method, a pool of degenerate oligonucleotides that target specific genomic sites is cyclically introduced into various cells within the population, which generates diversity across the population. MAGE was used to modify twenty four genetic parts in the DXP pathway, including ribosomal binding sites and enzyme coding sequences for tuning expression levels and knocking out gene function. This strategy resulted in a fivefold increase in lycopene production in only three days, demonstrating a practical alternative to encoding pathways on plasmids that often require many iterations of testing and optimization.

Plasmid-based expression and chromosomal integration are the two common vehicles for implementing synthetic metabolic pathways and each method has its advantages, described in an excellent review on gene-expression tools 
TABLE 1: Compilation of biological controllers.

\begin{tabular}{ll}
\hline Biological process & Genetic part or mechanism \\
\hline $\begin{array}{l}\text { Replication } \\
\text { Transcription } \\
\text { Initation }\end{array}$ & Replication origin (copy number) [34] \\
$\begin{array}{l}\text { Elongation } \\
\text { Rrocessing } \\
\text { enhancers, operator-regulator pairs [39], methylation }\end{array}$ \\
Rosttranscription \\
$\begin{array}{l}\text { Regradation } \\
\text { Translation }\end{array}$ \\
$\begin{array}{l}\text { Splice sites, polyA sites, TIGRs [41] } \\
\text { Initiation }\end{array}$ \\
$\begin{array}{l}\text { TIGRs [41], stabilizers, RNase sites [42] } \\
\text { Processing }\end{array}$ \\
$\begin{array}{l}\text { Ribosome-RBS pairs [43], Riboregulators, Riboswitches, Ribozymes, Antisense RNA, MicroRNA, } \\
\text { Small interfering RNA. For a review on RNA parts see [44]. }\end{array}$ \\
$\begin{array}{l}\text { Degradation } \\
\text { Localization }\end{array}$ & Codon bias, rare codons, stop codon-supressors pairs [15] \\
\hline
\end{tabular}

[48]. Recently, a new long-term expression method called chemically inducible chromosomal evolution (CIChE) was presented as an alternative to existing methods [49]. CIChE avoids complications associated with plasmid replication and segregation, and can be used to integrate multiple copies of genes (up to 40 copies demonstrated) into the genome. These techniques, coupled with in vivo strategies for assembling genetic parts into systems encoding metabolic pathways [50-52] and large-scale (several hundred kilobases) in vitro assembly methods [53], will provide technical platforms for the rapid synthesis of parts and subsequent pathways. On a larger scale, bacterial genome transplantation and the chemical synthesis of a natural bacterial genome have been demonstrated $[54,55]$.

\section{Analysis}

Cells are not unlike computers in that they process input information to realize output functions. A cell's genome can therefore be thought of as an operating system (OS) and, similarly, engineered operons encoding metabolic pathways as individual programs that are built from wellcharacterized, modular parts (the equivalent of a function in computer science terms). A twist on this analogy is that there already exists a large pool of parts and pathways in natural and synthetic biological systems and we are collectively learning their biological function through biochemical and bioinformatic analyses. Therefore, before metabolic pathways can be rationally designed and constructed, the genetic parts from which the pathways are built must be carefully characterized.

4.1. Parts. With the capability to synthesize biological parts, it is possible to use molecular-level controls to study and analyze each part [56]. One of the major stumbling blocks for the development of well-characertized parts is figuring out exactly what parameters should be measured and how. Although made from five basic parts, the well-characterized BioBrick signaling device BBa_F2620 developed by Canton et al. provided the first example of what can be measured and a possible standard documentation format-a datasheet (see Canton et al. [57] for a detailed example of a datasheet). The device's performance characteristics, including input compatibility, reliability, and transcriptional output demand, are neatly summarized. Datasheets may facilitate rapid widespread adoption of synthetic biological parts, devices, and systems if they include relevant information that is available to the community [58].

Recent work toward characterizing promoters demonstrates the utility of measuring the relative strengths of promoters in a library as they are built [12]. A separate effort by Kelly et al. has proposed a promoter reference standard, a fluoresense-dependent measurement kit and a measurement unit used to report promoter activity as an attempt to normalize absolute measurements taken by various instruments under various conditions [59]. The conceptual framework developed for promoter measurement could easily be applied to other genetic parts such as RBSs and transcriptional terminators. However, these tools will only be beneficial if they are supported and adopted by the community.

4.2. Pathways. Although well-characterized parts will drastically improve metabolic engineering efforts, there will always be a need for debugging tools and troubleshooting methods. Systems biology provides a framework for analyzing metabolic pathway performance and identifying possible solutions. Integrating high-throughput global measurements (i.e., transcriptomics, proteomics, metabolomics, and fluxomics) and computational tools (see Section 5) offers unprecedented understanding of how engineered pathways affect cell physiology and vice versa across the entire cell [60]. This kind of analysis can be used to identify bottlenecks in the 
pathway as well as detrimental side effects that may not have been obvious at the initial design stage [61].

For example, Kizer et al. used transciptional and metabolite profiling to determine the biochemical interactions of an engineered pathway and the endogenous metabolic network [62]. In this work, they were not only able to identify the bottleneck that resulted in toxic accumulation of a pathway intermediate, but also the mechanism by which the accumulated metabolite inhibits cellular growthand a way to overcome the problem. Using a system biology analytical approach, metabolic engineers are able to intelligently modulate synthetic pathway behavior if the measured performance does not meet specifications. Furthermore, the synthetic biology framework should enable the rational forward-engineering of pathways in a more plugand-play fashion and significantly decrease the time spent on debugging the genetic programs used to encode metabolic function.

\section{Modeling}

Highly complex electrical circuits are able to be designed with ease because of the existence of computer-aided design (CAD) software. This technology is dependent on the high-fidelity virtual representation of physical circuit componentry, which necessitates an accurate mathematical model of each component (e.g., transistors, capacitors) based on a deep understanding of the physics involved. Once the fundamental physics of genetic part behavior is understood, then parts can be designed to be insulated from each other. Subsequently, abstract design based on standard biological models would be possible, allowing CAD of intricate metabolic pathways and regulatory networks. While this is the ideal, there are a variety of modeling approaches being applied to biology that have various strengths and weaknesses leaving a consolidated, standard modeling approach something to strive for in the future.

5.1. Parts. At the part-level, it is possible to develop highly detailed, quantitative, dynamic models when considering a single or small number of parts. The software tool ProMoT was developed for modeling parts with ordinary differential equations (ODEs) and enabling interfacing by quantifying the exchange of biological signals (i.e., RNA polymerases, ribosomes, transcription factors and environmental stimuli) between parts [63]. In a similar spirit, SBML- and CellML-based models of genetic parts and well-characterized modules have been proposed for establishing a registry for standard biological models $[64,65]$. Standard, quantitative models of genetic parts would allow biological engineers to seamlessly integrate the design process with modeling, enabling simulation of engineered systems prior to actual construction (see design/model loop in Figure 1).

5.2. Pathways. As the scale of the system to be modeled increases from a single pathway to all pathways within an organism, more attention must be given to selecting a modeling approach as the quality of simulation results can vary simply due to the practical limitations imposed by the increasing number of variables. It is anticipated that as synthetic biology and modeling methods progress, the line between design and modeling will be blurred. This will condense the workflow into the common design-buildtest engineering cycle. A variety of $\mathrm{CAD}$ tools are emerging to make this happen $[66,67]$. Other computational tools are being developed to make novel pathway design a highthroughput process. From Metabolite to Metabolite (FMM) is a web-based tool that constructs a variety of possible enzymatic pathways from an input metabolite to an output metabolite, both of which are provided by the user [68]. This saves a large amount of time spent searching through the databases for relevant enzymes used in engineered pathways. Although not automated, the Retro-Biosynthesis Tool (ReBiT) provides access to a searchable database of enzymecatalyzed reactions categorized by the relevant functional groups of the reactants and products involved in the reaction [69]. ReBiT returns a list of enzymes capable of consuming or generating any user-defined functional group, which is particularly helpful for designing novel metabolic pathways (i.e., nonnatural).

Although these tools will facilitate the design of engineered systems, they will also need to take into account connectivity with the existing cellular network. At this point, even the most well-understood model microorganisms like E. coli and S. cerevisiae are far from being completely modeled [70, 71]. Therefore, interfacing engineered pathways with natural networks remains a difficult task [72]. However, existing metabolic models, when combined with powerful CAD tools to develop novel biological systems, will streamline the metabolic engineering process. Current genome-scale metabolic models are developed by deriving reaction list from genome annotation, refined using literature information and experimental data, and probed using constraint-based algorithms such as flux balance analysis (FBA) [73]. This modeling approach should enable decisionmaking when it comes to choosing an appropriate microbe to host a particular synthetic pathway and provide a means of predicting the in-context consequences of heterologous expression.

\section{Perspectives}

Genetically programming microbes to perform desired tasks has great potential to transform modern biotechnology. Significant progress has already been made in implementing novel biological functionality in microorganisms, including complex sensing/actuation algorithms and genetic circuits that count [74-77]. These advances have implications for metabolic engineering. For example, genetic circuits have been engineered to control the transcription of an operon that encodes enzymes and machinery necessary to manufacture and secrete recombinant spider silk monomers in Salmonella [78]. As progress is made in synthetic biology to develop well-characterized parts and increasingly complex devices, the design, modeling, synthesis, and analysis of synthetic metabolic pathways will become easier. 
In metabolic engineering, there is an implicit view of cells as chemical factories. As such, it can be useful to borrow conceptual frameworks from chemical engineering. Chemical plants are built from components that generally fall into one of three classes: unit operations (e.g., mixers, reactors, separators), connectors (pipes, tanks), and process controllers (measurement instruments, valves). These component classes exist within cells, but need to be further refined so that they can be used at the level of sophistication found in a chemical plant. In addition to advancing metabolic control through regulation of gene expression (e.g., engineered promoters, tunable intergenic regions [41]) and enzyme colocalization (modular protein scaffolds, or perhaps synthetic organelles), the enzymes themselves (i.e., specificity, activity, and allostery) can be modulated through directed evolution $[79,80]$ or perhaps designed/redesigned computationally. In fact, a recent study showed that swapping catalytic domains from three fungal cellulases can produce chimeras with superior properties [81]. Furthermore, engineered enzymes with novel catalytic properties would enable nonnatural metabolic pathway implementation. However, rationally engineering enzymes remain difficult and must be conducted on a case-by-case basis.

Therefore, it would be advantageous for the metabolic engineering community to work within the synthetic biology framework and focus on generating and populating families of genetic parts that are scalable, measured, and modeled. The simultaneous construction and characterization of parts will lead to model-based design of pathways in the future, just as complex electrical circuits are built in silico from well-modeled components. Highthroughput screening technologies have become powerful platforms for quantifying component performance of combinatorially-generated libraries [41]. Likewise, highthroughput measurements provide insight about evolved systems that have adapted to meet performance criteria [82]. These approaches could be very useful in identifying the genetic basis of the biological solution that evolution has provided, a strategy commonly referred to as inverse metabolic engineering [83]. The interplay of rational, combinatorial, and evolutionary approaches is crucial to the continued elucidation of biological design principles, which allow further forward-engineering of biological systems [84].

In order to achieve the forward-engineering of systemslevel metabolism, the design, modeling, synthesis, and analysis stages must incorporate shared standards in order to efficiently work together. In particular, well-characterized, off-the-shelf genetic components and computational models of these components must be available for use within a standard biological CAD tool. As models of components and modules develop, metabolic engineers will be able to move away from using the equivalent of biological machine code (DNA bases: ATCGs) for design to using compiled biological programs (genes and genetic circuits) that will enable faster pathway design by reusing pieces of previously optimized pathways. Similarly, characterization methods and standard measurement units must be agreed on so that parts are

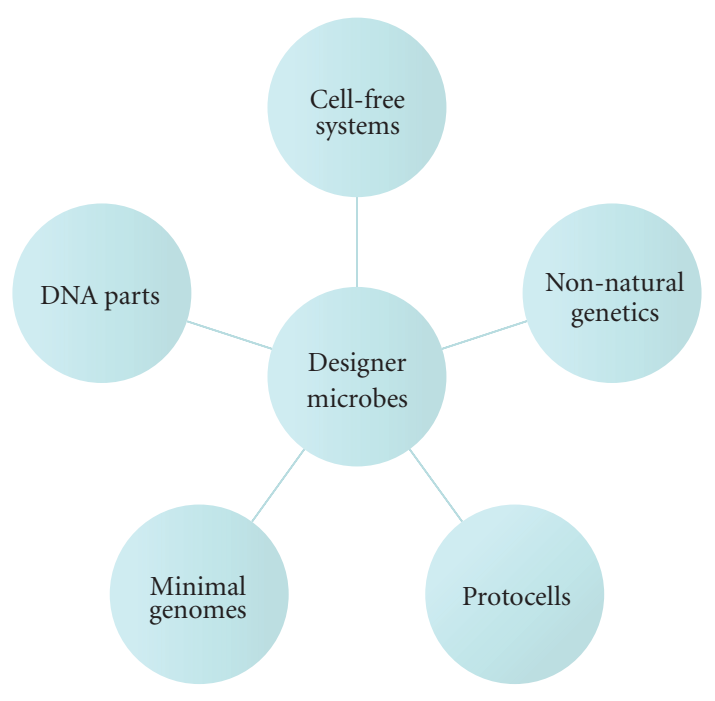

FIGURE 3: Convergence of the different realms of synthetic biology. In addition to efforts to refine and characterize genetic parts based on DNA, research on expanding the genetic code and implementing biological processes in cell-free systems will likely integrate with metabolic engineering projects in the future. Genome engineering and synthetic genomics will enable the manufacture of customizable minimal genomes. Alternatively, protocell engineering may provide a non-biological chassis to house synthetic chemistry.

characterized as they are made in a way that is useful to the community.

As already envisioned by a number of people [56, 61], integration of synthetic biology and systems biology will likely occur as both fields grow, eventually leading to tools that can allow for true cellular-scale network and pathway engineering. In many respects the fields of synthetic biology and systems biology are perfect complements to each other in regard to outlook and approach as we move toward wholecell engineering. While some overlap already exists between the two fields, synthetic biology currently has significant strengths in the steps of biological design and synthesis whereas systems biology has strengths in analysis and modeling (Figure 1). To properly integrate the detailed, dynamic, small-network components of synthetic biology with the large-scale analyses and algorithms of systems biology, it will be necessary to ensure that work in each field is compatible with work in the other field. For example, large-scale systems biology models will need to be able to incorporate dynamic behavior demonstrated by constructed synthetic circuits, and synthetic circuits should be constructed and implemented to have minimal cross-talk with existing cellular components. One can imagine a time when design can happen at a largescale level using systems biology algorithms [85-87] followed by molecular-level design using synthetic biology tools, de novo synthesis of components and pathways, analysis of results using high-throughput systems biology methods, and a hybrid large-scale, dynamic model of the entire process.

As the field of synthetic biology matures, various branches of synthetic biology that were not discussed here 
will soon be relevant to implementing novel metabolism in microbes (Figure 3). For example, a streamlined chassis based on a minimal, refactored genome would simplify host/system interactions and potentially minimize the effect of the metabolic burden the exogenous pathway would place on the cell. Likewise, the concept of designer microbes with engineered genomes could be realized by the developing area of synthetic genomics, which is dependent on the continued improvement in DNA synthesis technology. In the near future, host strains that have been engineered to confer complex phenotypes (e.g., solvent tolerance) using techniques such global transcription machinery engineering (gTME) may provide excellent chassis for synthetic metabolic systems $[37,88]$. Even so, recent successes in synthetic biologyenabled metabolic pathway engineering in common lab strains are very encouraging [31, 89-93]. Furthermore, work done toward engineering microbial communities may provide an approach to industrial biosynthesis that is superior to monocultures due to increased diversity and flexibility [94]. The future of this field is extremely exciting and will likely include rapid prototyping, testing, and debugging of synthetic metabolism for the industrial-scale microbial production of a wide variety of natural and novel chemicals and materials.

\section{Acknowledgment}

The authors thank Julius B. Lucks for thoughtful discussions and input.

\section{References}

[1] J. W. Chin, "Modular approaches to expanding the functions of living matter," Nature Chemical Biology, vol. 2, no. 6, pp. 304-311, 2006.

[2] D. Endy, "Foundations for engineering biology," Nature, vol. 438, no. 7067, pp. 449-453, 2005.

[3] S. A. Benner and A. M. Sismour, "Synthetic biology," Nature Reviews Genetics, vol. 6, no. 7, pp. 533-543, 2005.

[4] T. S. Ham, S. K. Lee, J. D. Keasling, and A. P. Arkin, "Design and construction of a double inversion recombination switch for heritable sequential genetic memory," PLoS ONE, vol. 3, no. 7 , article e2815, 2008.

[5] J. D. Keasling, "Synthetic biology for synthetic chemistry," ACS Chemical Biology, vol. 3, no. 1, pp. 64-76, 2008.

[6] J. B. Lucks, L. Qi, W. R. Whitaker, and A. P. Arkin, "Toward scalable parts families for predictable design of biological circuits," Current Opinion in Microbiology, vol. 11, no. 6, pp. 567-573, 2008.

[7] T. Y. Kim, S. B. Sohn, H. U. Kim, and S. Y. Lee, "Strategies for systems-level metabolic engineering," Biotechnology Journal, vol. 3, no. 5, pp. 612-623, 2008.

[8] P. E. M. Purnick and R. Weiss, "The second wave of synthetic biology: from modules to systems," Nature Reviews Molecular Cell Biology, vol. 10, no. 6, pp. 410-422, 2009.

[9] E. Andrianantoandro, S. Basu, D. K. Karig, and R. Weiss, "Synthetic biology: new engineering rules for an emerging discipline," Molecular Systems Biology, vol. 2, Article ID 2006.0028, 14 pages, 2006.
[10] M. Welch, A. Villalobos, C. Gustafsson, and J. Minshull, "You're one in a googol: optimizing genes for protein expression," Journal of the Royal Society Interface, vol. 6, supplement 4, pp. S467-S476, 2009.

[11] R. P. Shetty, D. Endy, and T. F. Knight Jr., "Engineering BioBrick vectors from BioBrick parts," Journal of Biological Engineering, vol. 2, article 5, 2008.

[12] T. Ellis, X. Wang, and J. J. Collins, "Diversity-based, modelguided construction of synthetic gene networks with predicted functions," Nature Biotechnology, vol. 27, no. 5, pp. 465-471, 2009.

[13] H. M. Salis, E. A. Mirsky, and C. A. Voigt, "Automated design of synthetic ribosome binding sites to control protein expression," Nature Biotechnology, vol. 27, no. 10, pp. 946-950, 2009.

[14] T. S. Ham, S. K. Lee, J. D. Keasling, and A. P. Arkin, "A tightly regulated inducible expression system utilizing the fim inversion recombination switch," Biotechnology and Bioengineering, vol. 94, no. 1, pp. 1-4, 2006.

[15] J. C. Anderson, C. A. Voigt, and A. P. Arkin, "Environmental signal integration by a modular and gate," Molecular Systems Biology, vol. 3, article 133, 2007.

[16] F. J. Isaacs, D. J. Dwyer, C. Ding, D. D. Pervouchine, C. R. Cantor, and J. J. Collins, "Engineered riboregulators enable posttranscriptional control of gene expression," Nature Biotechnology, vol. 22, no. 7, pp. 841-847, 2004.

[17] M. N. Win and C. D. Smolke, "A modular and extensible RNA-based gene-regulatory platform for engineering cellular function," Proceedings of the National Academy of Sciences of the United States of America, vol. 104, no. 36, pp. 14283-14288, 2007.

[18] M. N. Win, J. C. Liang, and C. D. Smolke, "Frameworks for programming biological function through RNA parts and devices," Chemistry and Biology, vol. 16, no. 3, pp. 298-310, 2009.

[19] D. J. Pitera, C. J. Paddon, J. D. Newman, and J. D. Keasling, "Balancing a heterologous mevalonate pathway for improved isoprenoid production in Escherichia coli," Metabolic Engineering, vol. 9, no. 2, pp. 193-207, 2007.

[20] M. E. Wall, W. S. Hlavacek, and M. A. Savageau, "Design of gene circuits: lessons from bacteria," Nature Reviews Genetics, vol. 5, no. 1, pp. 34-42, 2004.

[21] M. Kærn, T. C. Elston, W. J. Blake, and J. J. Collins, "Stochasticity in gene expression: from theories to phenotypes," Nature Reviews Genetics, vol. 6, no. 6, pp. 451-464, 2005.

[22] A. Raj and A. van Oudenaarden, "Nature, nurture, or chance: stochastic gene expression and its consequences," Cell, vol. 135, no. 2, pp. 216-226, 2008.

[23] T. S. Gardner, C. R. Cantor, and J. J. Collins, "Construction of a genetic toggle switch in Escherichia coli," Nature, vol. 403, no. 6767, pp. 339-342, 2000.

[24] M. B. Elowitz and S. Leibier, "A synthetic oscillatory network of transcriptional regulators," Nature, vol. 403, no. 6767, pp. 335-338, 2000.

[25] T. E. Landrain, J. Carrera, B. Kirov, G. Rodrigo, and A. Jaramillo, "Modular model-based design for heterologous bioproduction in bacteria," Current Opinion in Biotechnology, vol. 20, no. 3, pp. 272-279, 2009.

[26] R. J. Conrado, J. D. Varner, and M. P. DeLisa, "Engineering the spatial organization of metabolic enzymes: mimicking nature's synergy," Current Opinion in Biotechnology, vol. 19, no. 5, pp. 492-499, 2008. 
[27] J. E. Dueber, G. C. Wu, G. R. Malmirchegini, et al., "Synthetic protein scaffolds provide modular control over metabolic flux," Nature Biotechnology, vol. 27, no. 8, pp. 753-759, 2009.

[28] C. A. Voigt, "Genetic parts to program bacteria," Current Opinion in Biotechnology, vol. 17, no. 5, pp. 548-557, 2006.

[29] M. R. Bennett and J. Hasty, "Overpowering the component problem," Nature Biotechnology, vol. 27, no. 5, pp. 450-451, 2009.

[30] J. H. Park, S. Y. Lee, T. Y. Kim, and H. U. Kim, "Application of systems biology for bioprocess development," Trends in Biotechnology, vol. 26, no. 8, pp. 404-412, 2008.

[31] T. S. Bayer, D. M. Widmaier, K. Temme, E. A. Mirsky, D. V. Santi, and C. A. Voigt, "Synthesis of methyl halides from biomass using engineered microbes," Journal of the American Chemical Society, vol. 131, no. 18, pp. 6508-6515, 2009.

[32] S. J. Reisinger, K. G. Patel, and D. V. Santi, "Total synthesis of multi-kilobase DNA sequences from oligonucleotides," Nature Protocols, vol. 1, no. 6, pp. 2596-2603, 2007.

[33] M. J. Czar, J. C. Anderson, J. S. Bader, and J. Peccoud, "Gene synthesis demystified," Trends in Biotechnology, vol. 27, no. 2, pp. 63-72, 2009.

[34] K. L. Jones, S.-W. Kim, and J. D Keasling, "Low-copy plasmids can perform as well as or better than high-copy plasmids for metabolic engineering of bacteria," Metabolic Engineering, vol. 2, no. 4, pp. 328-338, 2000.

[35] D. Klein-Marcuschamer, C. N. S. Santos, H. Yu, and G. Stephanopoulos, "Mutagenesis of the bacterial RNA polymerase alpha subunit for improvement of complex phenotypes," Applied and Environmental Microbiology, vol. 75, no. 9, pp. 2705-2711, 2009.

[36] T. Sera, "Zinc-finger-based artificial transcription factors and their applications," Advanced Drug Delivery Reviews, vol. 61, no. 7-8, pp. 513-526, 2009.

[37] H. Alper and G. Stephanopoulos, "Global transcription machinery engineering: a new approach for improving cellular phenotype," Metabolic Engineering, vol. 9, no. 3, pp. 258-267, 2007.

[38] H. Alper, C. Fischer, E. Nevoigt, and G. Stephanopoulos, "Tuning genetic control through promoter engineering," Proceedings of the National Academy of Sciences of the United States of America, vol. 102, no. 36, pp. 12678-12683, 2005.

[39] M. Krueger, O. Scholz, S. Wisshak, and W. Hillen, "Engineered Tet repressors with recognition specificity for the tetO-4C5G operator variant," Gene, vol. 404, no. 1-2, pp. 93-100, 2007.

[40] L. Du, R. Gao, and A. C. Forster, "Engineering multigene expression in vitro and in vivo with small terminators for $\mathrm{T} 7$ RNA polymerase," Biotechnology and Bioengineering, vol. 104, no. 6, pp. 1189-1196, 2009.

[41] B. F. Pfleger, D. J. Pitera, C. D. Smolke, and J. D. Keasling, "Combinatorial engineering of intergenic regions in operons tunes expression of multiple genes," Nature Biotechnology, vol. 24, no. 8, pp. 1027-1032, 2006.

[42] C. D. Smolke, T. A. Carrier, and J. D. Keasling, "Coordinated, differential expression of two genes through directed mRNA cleavage and stabilization by secondary structures," Applied and Environmental Microbiology, vol. 66, no. 12, pp. 53995405, 2000.

[43] O. Rackham and J. W. Chin, "Cellular logic with orthogonal ribosomes," Journal of the American Chemical Society, vol. 127, no. 50, pp. 17584-17585, 2005.

[44] F. J. Isaacs, D. J. Dwyer, and J. J. Collins, "RNA synthetic biology," Nature Biotechnology, vol. 24, no. 5, pp. 545-554, 2006.
[45] C. Engler, R. Gruetzner, R. Kandzia, and S. Marillonnet, "Golden gate shuffling: a one-pot DNA shuffling method based on type ils restriction enzymes," PLoS ONE, vol. 4, no. 5, article e5553, 2009.

[46] J. Quan and J. Tian, "Circular polymerase extension cloning of complex gene libraries and pathways," PLoS ONE, vol. 4, no. 7, article e6441, 2009.

[47] H. H. Wang, F. J. Isaacs, P. A. Carr, et al., "Programming cells by multiplex genome engineering and accelerated evolution," Nature, vol. 460, no. 7257, pp. 894-898, 2009.

[48] J. D. Keasling, "Gene-expression tools for the metabolic engineering of bacteria," Trends in Biotechnology, vol. 17, no. 11, pp. 452-460, 1999.

[49] K. E. J. Tyo, P. K. Ajikumar, and G. Stephanopoulos, "Stabilized gene duplication enables long-term selection-free heterologous pathway expression," Nature Biotechnology, vol. 27, no. 8, pp. 760-765, 2009.

[50] Z. Shao, H. Zhao, and H. Zhao, "DNA assembler, an in vivo genetic method for rapid construction of biochemical pathways," Nucleic Acids Research, vol. 37, no. 2, article e16, 2009.

[51] R. M. Q. Shanks, N. C. Caiazza, S. M. Hinsa, C. M. Toutain, and G. A. O’Toole, "Saccharomyces cerevisiae-based molecular tool kit for manipulation of genes from gramnegative bacteria," Applied and Environmental Microbiology, vol. 72, no. 7, pp. 5027-5036, 2006.

[52] D. G. Gibson, G. A. Benders, K. C. Axelrod, et al., "Onestep assembly in yeast of 25 overlapping DNA fragments to form a complete synthetic Mycoplasma genitalium genome," Proceedings of the National Academy of Sciences of the United States of America, vol. 105, no. 51, pp. 20404-20409, 2008.

[53] D. G. Gibson, L. Young, R.-Y. Chuang, J. C. Venter, C. A. Hutchison, and H. O. Smith, "Enzymatic assembly of DNA molecules up to several hundred kilobases," Nature Methods, vol. 6, no. 5, pp. 343-345, 2009.

[54] C. Lartigue, J. I. Glass, N. Alperovich, et al., "Genome transplantation in bacteria: changing one species to another," Science, vol. 317, no. 5838, pp. 632-638, 2007.

[55] D. G. Gibson, G. A. Benders, C. Andrews-Pfannkoch, et al., "Complete chemical synthesis, assembly, and cloning of a Mycoplasma genitalium genome," Science, vol. 319, no. 5867, pp. 1215-1220, 2008.

[56] C. L. Barrett, T. Y. Kim, H. U. Kim, B. Ø. Palsson, and S. Y. Lee, "Systems biology as a foundation for genome-scale synthetic biology," Current Opinion in Biotechnology, vol. 17, no. 5, pp. 488-492, 2006.

[57] B. Canton, A. Labno, and D. Endy, "Refinement and standardization of synthetic biological parts and devices," Nature Biotechnology, vol. 26, no. 7, pp. 787-793, 2008.

[58] A. Arkin, "Setting the standard in synthetic biology," Nature Biotechnology, vol. 26, no. 7, pp. 771-774, 2008.

[59] J. R. Kelly, A. J. Rubin, J. H. Davis, et al., "Measuring the activity of BioBrick promoters using an in vivo reference standard," Journal of Biological Engineering, vol. 3, article 4, 2009.

[60] S. J. Park, S. Y. Lee, J. Cho, et al., "Global physiological understanding and metabolic engineering of microorganisms based on omics studies," Applied Microbiology and Biotechnology, vol. 68, no. 5, pp. 567-579, 2005.

[61] A. Mukhopadhyay, A. M. Redding, B. J. Rutherford, and J. D. Keasling, "Importance of systems biology in engineering microbes for biofuel production," Current Opinion in Biotechnology, vol. 19, no. 3, pp. 228-234, 2008. 
[62] L. Kizer, D. J. Pitera, B. F. Pfleger, and J. D. Keasling, "Application of functional genomics to pathway optimization for increased isoprenoid production," Applied and Environmental Microbiology, vol. 74, no. 10, pp. 3229-3241, 2008.

[63] M. A. Marchisio and J. Stelling, "Computational design of synthetic gene circuits with composable parts," Bioinformatics, vol. 24, no. 17, pp. 1903-1910, 2008.

[64] L. Endler, N. Rodriguez, N. Juty, et al., "Designing and encoding models for synthetic biology," Journal of the Royal Society Interface, vol. 6, supplement 4, pp. S405-S417, 2009.

[65] V. Rouilly, B. Canton, P. Nielsen, and R. Kitney, "Registry of BioBricks models using CellML," BMC Systems Biology, vol. 1, article 79, 2007.

[66] M. J. Czar, Y. Cai, and J. Peccoud, "Writing DNA with GenoCADTM," Nucleic Acids Research, vol. 37, supplement 2, pp. W40-W47, 2009.

[67] J. A. Goler, B. W. Bramlett, and J. Peccoud, "Genetic design: rising above the sequence," Trends in Biotechnology, vol. 26, no. 10, pp. 538-544, 2008.

[68] C.-H. Chou, W.-C. Chang, C.-M. Chiu, C.-C. Huang, and H.D. Huang, "FMM: a web server for metabolic pathway reconstruction and comparative analysis," Nucleic Acids Research, vol. 37, supplement 2, pp. W129-W134, 2009.

[69] K. L. J. Prather and C. H. Martin, "De novo biosynthetic pathways: rational design of microbial chemical factories," Current Opinion in Biotechnology, vol. 19, no. 5, pp. 468-474, 2008.

[70] D. W. Selinger, M. A. Wright, and G. M. Church, "On the complete determination of biological systems," Trends in Biotechnology, vol. 21, no. 6, pp. 251-254, 2003.

[71] D. J. Dwyer, M. A. Kohanski, and J. J. Collins, "Networking opportunities for bacteria," Cell, vol. 135, no. 7, pp. 11531156, 2008.

[72] H. Kobayashi, M. Kærn, M. Araki, et al., "Programmable cells: interfacing natural and engineered gene networks," Proceedings of the National Academy of Sciences of the United States of America, vol. 101, no. 22, pp. 8414-8419, 2004.

[73] J. M. Park, T. Y. Kim, and S. Y. Lee, "Constraints-based genome-scale metabolic simulation for systems metabolic engineering," Biotechnology Advances, vol. 27, no. 6, pp. 979988, 2009.

[74] J. C. Anderson, E. J. Clarke, A. P. Arkin, and C. A. Voigt, "Environmentally controlled invasion of cancer cells by engineered bacteria," Journal of Molecular Biology, vol. 355, no. 4, pp. 619 627, 2006.

[75] J. J. Tabor, H. M. Salis, Z. B. Simpson, et al., "A synthetic genetic edge detection program," Cell, vol. 137, no. 7, pp. 1272-1281, 2009.

[76] A. E. Friedland, T. K. Lu, X. Wang, D. Shi, G. Church, and J. J. Collins, "Synthetic gene networks that count," Science, vol. 324, no. 5931, pp. 1199-1202, 2009.

[77] A. Levskaya, O. D. Weiner, W. A. Lim, and C. A. Voigt, "Spatiotemporal control of cell signalling using a lightswitchable protein interaction," Nature, vol. 461, no. 7266, pp. 997-1001, 2009.

[78] D. M. Widmaier, D. Tullman-Ercek, E. A. Mirsky, et al., "Engineering the Salmonella type III secretion system to export spider silk monomers," Molecular Systems Biology, vol. 5, article 309, 2009.

[79] Y. Yoshikuni, J. A. Dietrich, F. F. Nowroozi, P. C. Babbitt, and J. D. Keasling, "Redesigning enzymes based on adaptive evolution for optimal function in synthetic metabolic pathways," Chemistry and Biology, vol. 15, no. 6, pp. 607-618, 2008.
[80] J. D. Bloom, M. M. Meyer, P. Meinhold, C. R. Otey, D. MacMillan, and F. H. Arnold, "Evolving strategies for enzyme engineering," Current Opinion in Structural Biology, vol. 15, no. 4, pp. 447-452, 2005.

[81] P. Heinzelman, C. D. Snow, I. Wu, et al., "A family of thermostable fungal cellulases created by structure-guided recombination," Proceedings of the National Academy of Sciences of the United States of America, vol. 106, no. 14, pp. 56105615, 2009.

[82] S. S. Fong, A. P. Burgard, C. D. Herring, et al., "In silico design and adaptive evolution of Escherichia coli for production of lactic acid," Biotechnology and Bioengineering, vol. 91, no. 5, pp. 643-648, 2005.

[83] C. N. S. Santos and G. Stephanopoulos, "Combinatorial engineering of microbes for optimizing cellular phenotype," Current Opinion in Chemical Biology, vol. 12, no. 2, pp. 168176, 2008.

[84] L. Rohlin, M.-K. Oh, and J. C. Liao, "Microbial pathway engineering for industrial processes: evolution, combinatorial biosynthesis and rational design," Current Opinion in Microbiology, vol. 4, no. 3, pp. 330-335, 2001.

[85] A. P. Burgard, P. Pharkya, and C. D. Maranas, "Optknock: a bilevel programming framework for identifying gene knockout strategies for microbial strain optimization," Biotechnology and Bioengineering, vol. 84, no. 6, pp. 647-657, 2003.

[86] P. Pharkya, A. P. Burgard, and C. D. Maranas, "OptStrain: a computational framework for redesign of microbial production systems," Genome Research, vol. 14, no. 11, pp. 2367-2376, 2004.

[87] D. S. Lun, G. Rockwell, N. J. Guido, et al., "Large-scale identification of genetic design strategies using local search," Molecular Systems Biology, vol. 5, article 296, 2009.

[88] J. R. Warner, R. Patnaik, and R. T. Gill, "Genomics enabled approaches in strain engineering," Current Opinion in Microbiology, vol. 12, no. 3, pp. 223-230, 2009.

[89] K. M. Hawkins and C. D. Smolke, "Production of benzylisoquinoline alkaloids in Saccharomyces cerevisiae," Nature Chemical Biology, vol. 4, no. 9, pp. 564-573, 2008.

[90] T. S. Moon, S.-H. Yoon, A. M. Lanza, J. D. Roy-Mayhew, and K. L. J. Prather, "Production of glucaric acid from a synthetic pathway in recombinant Escherichia coli," Applied and Environmental Microbiology, vol. 75, no. 3, pp. 589-595, 2009.

[91] K. Zhang, M. R. Sawaya, D. S. Eisenberg, and J. C. Liao, "Expanding metabolism for biosynthesis of nonnatural alcohols," Proceedings of the National Academy of Sciences of the United States of America, vol. 105, no. 52, pp. 20653-20658, 2008.

[92] V. J. J. Martin, D. J. Piteral, S. T. Withers, J. D. Newman, and J. D. Keasling, "Engineering a mevalonate pathway in Escherichia coli for production of terpenoids," Nature Biotechnology, vol. 21, no. 7, pp. 796-802, 2003.

[93] D.-K. Ro, E. M. Paradise, M. Quellet, et al., "Production of the antimalarial drug precursor artemisinic acid in engineered yeast," Nature, vol. 440, no. 7086, pp. 940-943, 2006.

[94] K. Brenner, L. You, and F. H. Arnold, "Engineering microbial consortia: a new frontier in synthetic biology," Trends in Biotechnology, vol. 26, no. 9, pp. 483-489, 2008. 

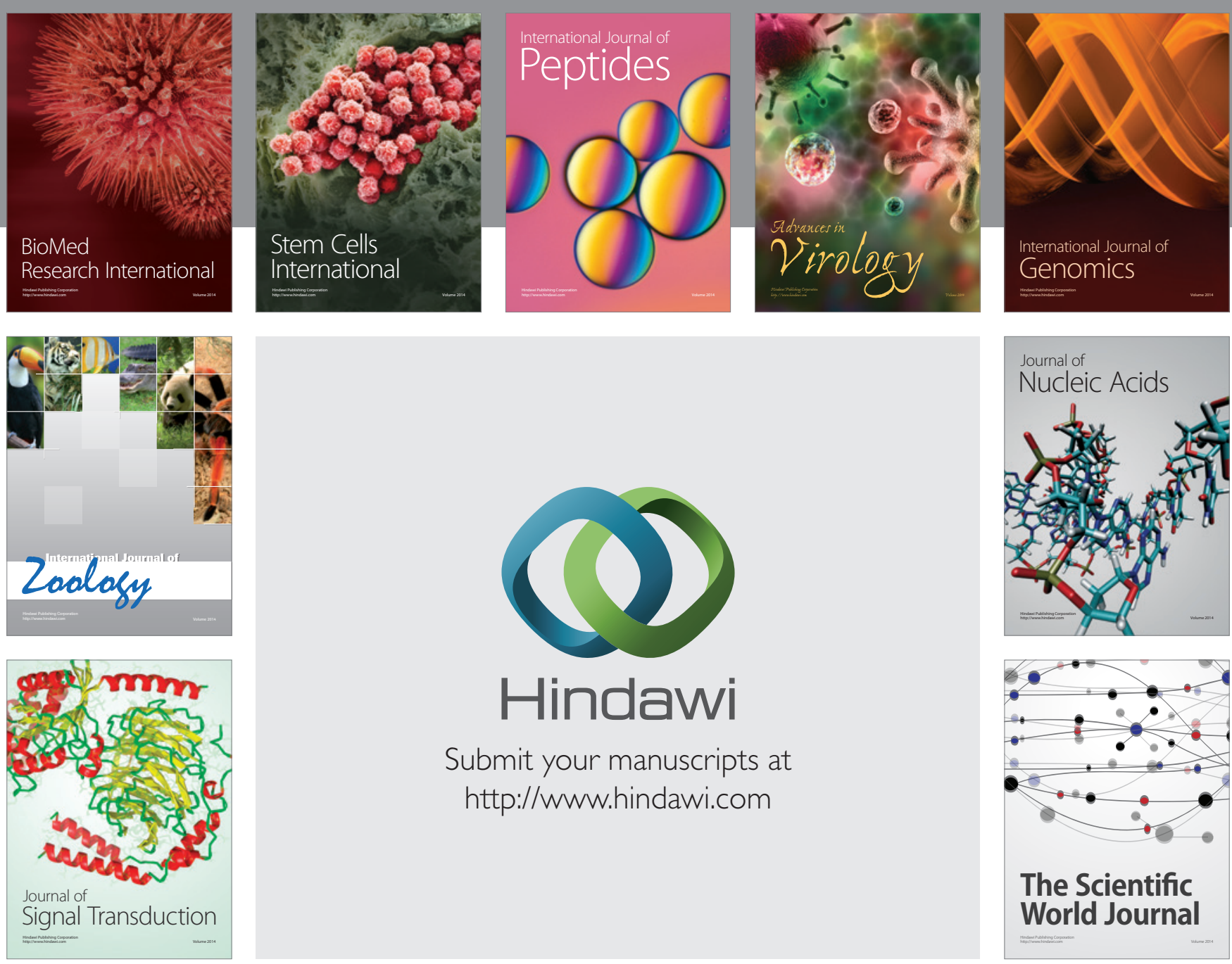

Submit your manuscripts at

http://www.hindawi.com
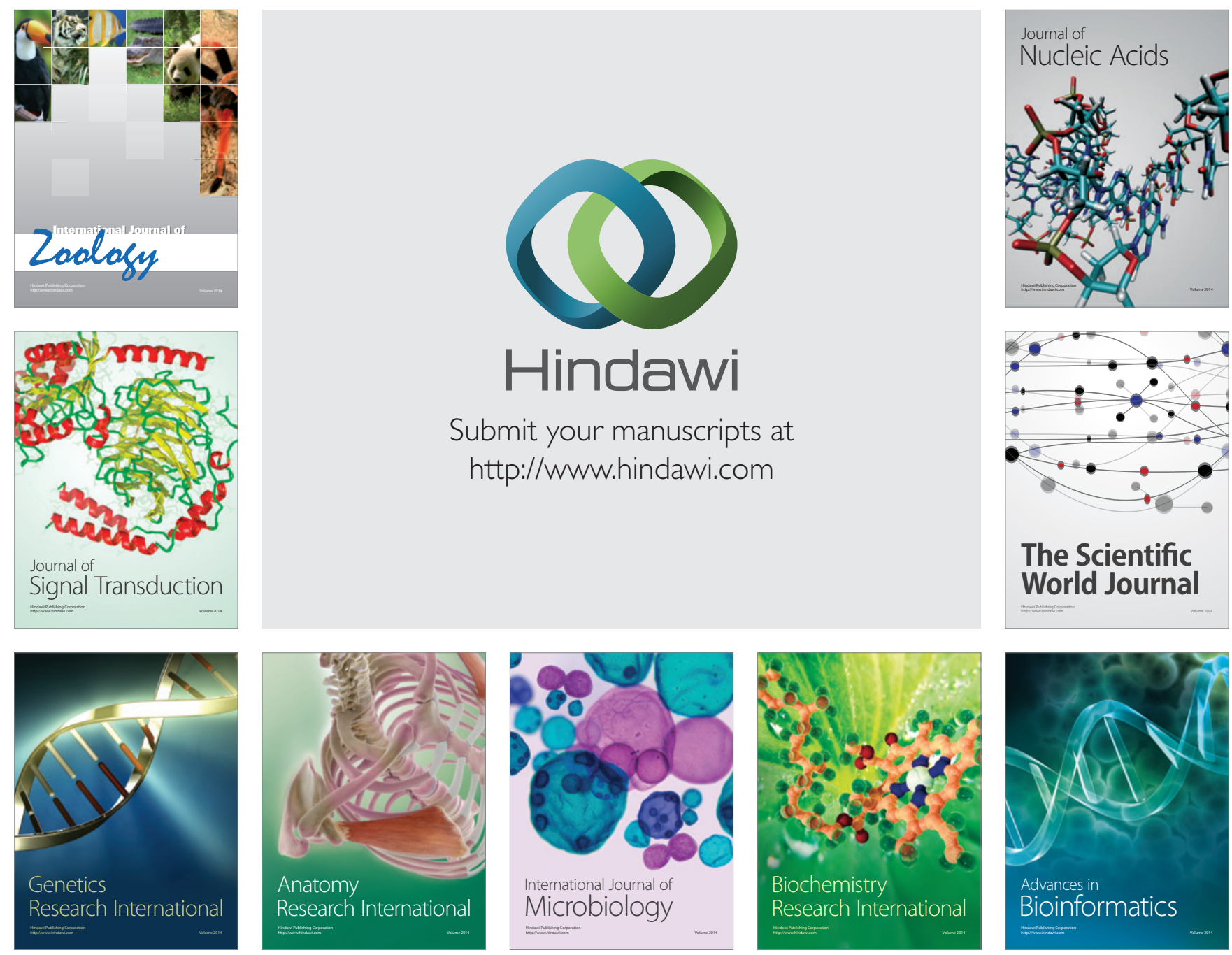

The Scientific World Journal
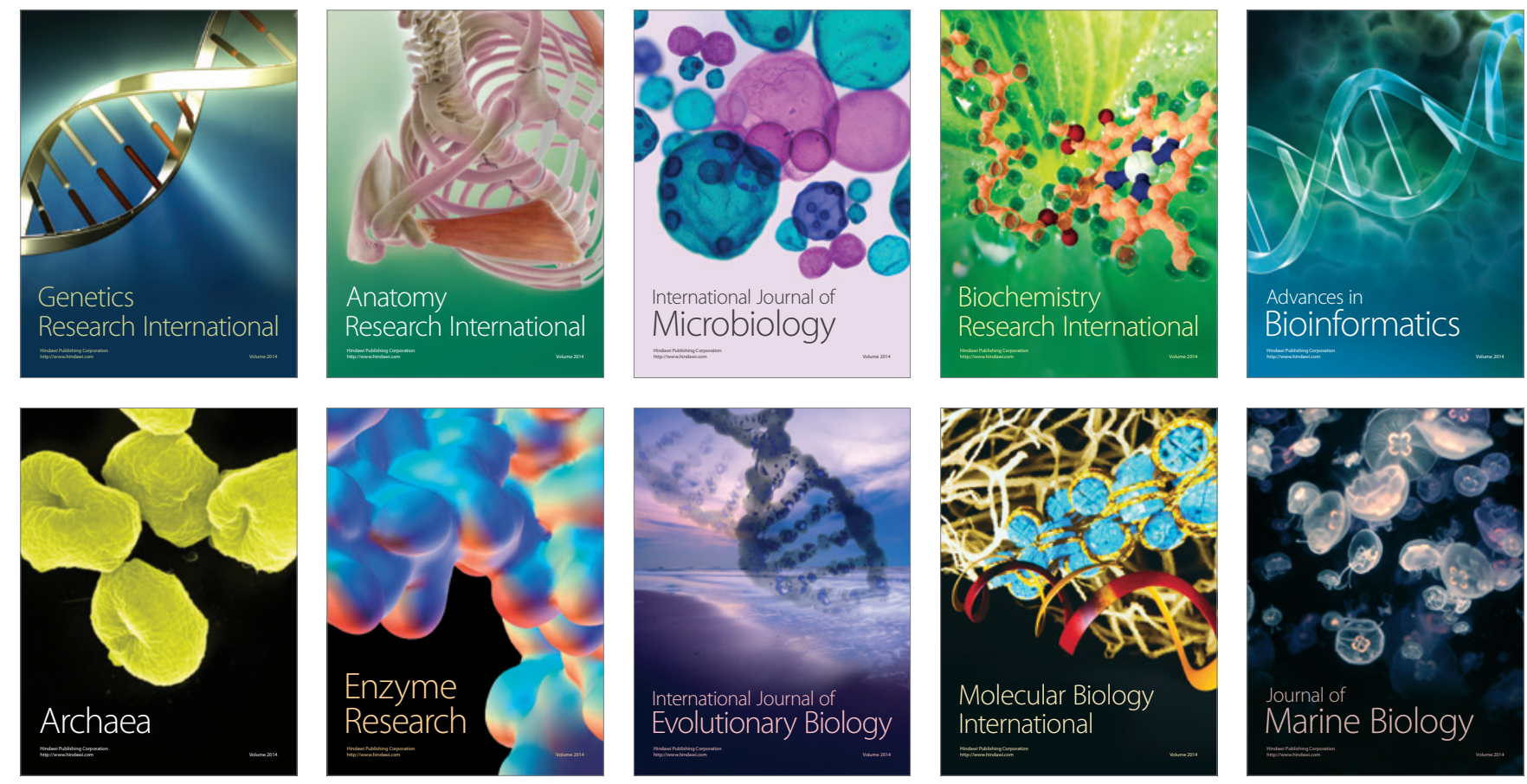